\title{
In-plane three-step needle insertion technique for ultrasound- guided continuous femoral nerve block after total knee arthroplasty : a retrospective review of 488 cases
}

\author{
Hyeon Ju Shin, Jung Sub Soh, Hyong Hwan Lim, Bumjoon Joo, \\ Hye Won Lee, and Hae Ja Lim \\ Department of Anesthesiology and Pain Medicine, Korea University College of Medicine, Seoul, Korea
}

\begin{abstract}
Background: Continuous femoral nerve block (CFNB) improves postoperative analgesia after total knee arthroplasty (TKA). The aim of this study was to investigate the clinical efficacy and complications of our in-plane three-step needle insertion technique that was devised to reduce the risk of direct femoral nerve injury during CFNB in anesthetized patients.

Methods: This retrospective study included 488 patients who had undergone TKA. Ultrasound (US)-guided CFNB was performed under general or spinal anesthesia using an in-plane, three-step needle insertion technique. The success rate and difficulties of catheter placement, clinical efficacy of analgesia, and complications were recorded.

Results: Femoral catheters were placed with a 100\% success rate. In 488 patients, real-time US imaging revealed easy separation of the fascia iliaca and the femoral nerve following injection of local anesthetic through a Tuohy needle. Verbal numerical rating scale pain scores $(0-10)$ were $2.0 \pm 1.2,3.5 \pm 1.9,3.2 \pm 1.7,2.9 \pm 1.3$, and $2.5 \pm 1.1$ at 1, 6, 12, 24 and $48 \mathrm{~h}$ postoperatively. No femoral hematoma, femoral abscess, or neurologic complications, including paresthesia or neurologic deficits, were observed during the 8-week follow-up period.

Conclusions: This retrospective study suggests that an in-plane three-step needle insertion technique for CFNB may reduce the risk of femoral nerve injury in anesthetized patients.
\end{abstract}

Key Words: Arthroplasty, Catheters, Femoral nerve, Knee, Ultrasonography.

Corresponding author: Hyeon Ju Shin, M.D., Ph.D.

Department of Anesthesiology and Pain Medicine, Korea University College of Medicine, 73, Inchon-ro, Seongbuk-gu, Seoul 02841, Korea

Tel: 82-2-920-5632, Fax: 82-2-929-2936, Email: may335@naver.com

ORCID: http://orcid.org/0000-0003-3150-6840

It was presented The 35th Annual European Society of Regional Anesthesia \& Pain Therapy Congress, September 2016, MECC Maastricht, Maastricht, Netherlands.

Received: April 7, 2016. Revised: May 13, 2016 (1st); June 14, 2016 (2nd); June 23, 2016 (3rd). Accepted: June 24, 2016.

Korean J Anesthesiol 2016 December 69(6): 587-591

https://doi.org/10.4097/kjae.2016.69.6.587

(c) This is an open-access article distributed under the terms of the Creative Commons Attribution Non-Commercial License (http://creativecommons.org/ licenses/by-nc/4.0/), which permits unrestricted non-commercial use, distribution, and reproduction in any medium, provided the original work is properly cited. 


\section{Introduction}

Continuous femoral nerve block (CFNB) is an effective analgesic technique for moderate to severe postoperative pain induced by total knee arthroplasty (TKA) [1,2]. The femoral nerve is covered by the fascia iliaca and separated from the femoral artery and femoral vein by the femoral sheath [3]. Therefore, for successful and safe placement of a femoral catheter, an 18- gauge (G) Tuohy needle should be used to penetrate the fascia iliaca at the lateral part of the femoral nerve, and a perineural space should be created via the injection of a local anesthetic beneath the fascia iliaca. Using this approach, the catheter can then be placed within the perineural space, thereby avoiding direct femoral nerve injury.

The performance of regional blockade in anesthetized patients may increase the risk of postoperative neurologic complications. Anesthetized patients are unable to respond to painful stimuli and paresthesia during needle or catheter placement and injection of local anesthetic [4]. However, most children who undergo regional anesthetic procedures are either heavily sedated or under general anesthesia $[5,6]$, and performing these blocks on anesthetized patients has several benefits, including greater patient acceptance, reduced impact on the operating list, and greater ease of performance [4].

Ultrasound (US) guidance provides the ability to visualize the needle-to-nerve relationship and the spread of a local anesthetic in real time, and may reduce the risk of neurologic injury [7]. In the current study, we evaluated the clinical outcome and complications of US-guided femoral catheter placement using our in-plane three-step needle insertion technique that was devised to reduce the risk of direct femoral nerve injury for TKA in 488 anesthetized patients.

\section{Materials and Methods}

After receiving ethics committee approval at Korea University College of Medicine (Seoul, South Korea), we retrospectively reviewed anesthesia charts and medical records of all patients with an American Society of Anesthesiologists physical status of 1-3 who received US-guided CFNB for TKA between October 2010 and December 2015. Of the 488 patients who were identified, 190 patients had received the US-guided CFNB under general anesthesia, while 298 patients received the US-guided CFNB under spinal anesthesia after TKA. All CFNB procedures were performed by an expert regional anesthesiologist (H.J. Shin).

In the operating room, standard noninvasive monitoring including electrocardiogram, blood pressure measurement, and pulse oximetry was performed. Patients received fentanyl $50 \mu \mathrm{g}$ and midazolam 1-2 mg intravenously for anxiolytic purposes, while remaining responsive to verbal commands.
General anesthesia was induced with propofol $(2 \mathrm{mg} / \mathrm{kg})$ and fentanyl $50 \mu \mathrm{g}$. Rocuronium $(0.6 \mathrm{mg} / \mathrm{kg})$ was administered to facilitate endotracheal intubation with a cuffed tube. Anesthesia was maintained with $2 \%$ sevoflurane, and CFNB was performed after the surgery and before extubation. General anesthesia was reversed with pyridostigmine and glycopyrrolate.

In the patients undergoing spinal anesthesia, spinal anesthesia was performed at the L3-4 or L4-5 intervertebral space with the patient in the lateral decubitus position, operated side down, using a 23- or 24-G Quincke needle. In all patients, hyperbaric bupivacaine hydrochloride 0.5\% (Marcaine Spinal Heavy ${ }^{\circledR}$ AstraZeneca, London, UK), 8-12 mg, was injected in the intrathecal space. CFNB was performed after surgery.

\section{Ultrasound technique}

CFNB was performed with patients in the supine position. After the skin was sterilized, a Sonosite M-Turbo (Sonosite, Bothell, WA, USA) 6-13 MHz linear high frequency US probe covered with a sterile vinyl cover and gel was placed at the level of the inguinal crease, and a short-axis US of the femoral nerve was obtained.

The skin was infiltrated with $2-3 \mathrm{ml} 1 \%$ lidocaine at the puncture site, approximately $2-3 \mathrm{~cm}$ lateral to the probe, and an 18-G 10-cm insulated Tuohy needle (Contiplex $\mathrm{B}^{\circledR}$, B. Braun, Melsungen, Germany) connected to the nerve stimulator (Stimuplex $^{\circledR}$, B. Braun, Melsungen, Germany) was inserted via an in-plane approach under US guidance.

The nerve stimulator was set to $0.5 \mathrm{~mA}$, with a pulse duration of $0.1 \mathrm{~ms}$ and stimulation frequency of $2 \mathrm{~Hz}$, and was used to check for contraction of the quadriceps femoris or sartorius muscle. The insertion point for the in-plane approach of the 18G Tuohy needle was $2-3 \mathrm{~cm}$ lateral to the US probe at the level of the inguinal crease.

\section{In-plane three-step needle insertion technique}

We performed CFNB under US guidance using an in-plane three-step needle insertion technique. First, the Tuohy needle was inserted to penetrate the fascia iliaca at the lateral part of the femoral nerve. Second, after penetration of the fascia iliaca, the needle tip was pulled back slightly and the angle of the needle shaft was decreased to near horizontal. Third, the needle was inserted into the space between the fascia iliaca and the femoral nerve and advanced until the tip was positioned beneath the fascia iliaca and the midpoint of the anterior surface of the femoral nerve (Fig. 1).

Ropivacaine $0.2-0.375 \%, 20 \mathrm{ml}$ was injected incrementally through the Tuohy needle after negative aspiration above the femoral nerve, to create a perineural space (Fig. 2). A 20-gauge 
catheter was threaded $2-3 \mathrm{~cm}$ beyond the needle tip and $10 \mathrm{ml}$ of normal saline was administered through the catheter after negative aspiration to confirm the position of the catheter. The catheter position was considered satisfactory if the spread of the normal saline through the catheter near the femoral nerve was

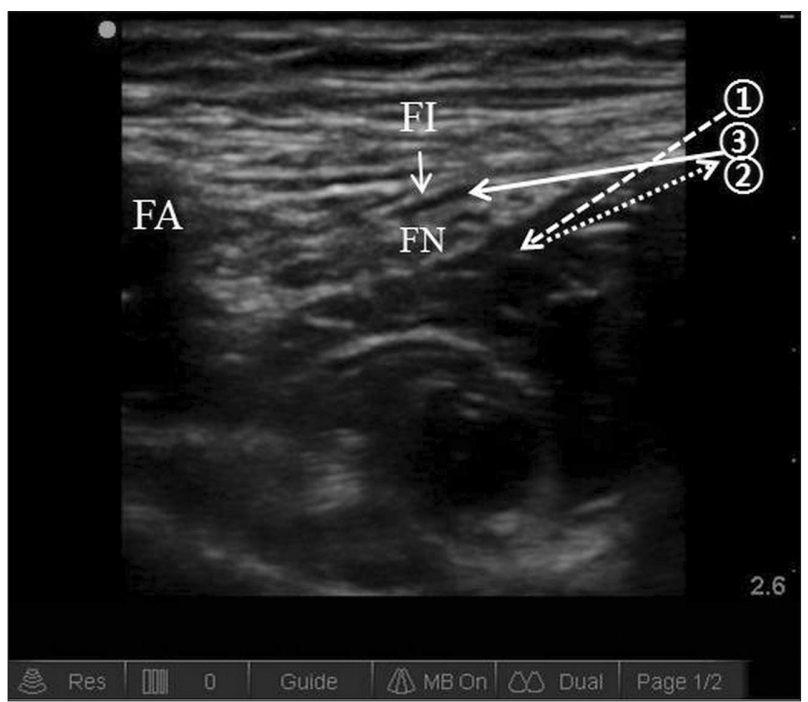

Fig. 1. Transverse sonogram of the femoral nerve and needle direction during the in-plane three-step needle insertion technique. Arrow 1 corresponds to the first step in which the Tuohy needle is inserted in order to penetrate the fascia iliaca at the lateral part of the femoral nerve. Step 2, indicated by arrow 2, involves the needle tip being pulled back in order to decrease the angle of the needle shaft. Arrow 3 shows the third step of the technique, in which the needle is inserted into the space between the fascia iliaca and the femoral nerve. FA: femoral artery, FI: fascia iliaca, FN: femoral nerve.

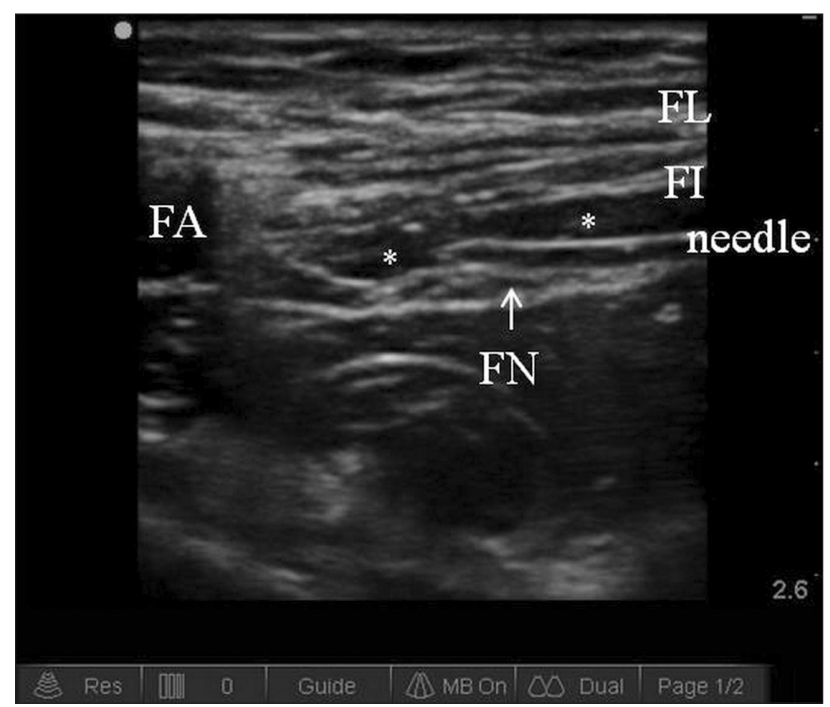

Fig. 2. Transverse sonogram of the femoral nerve after injection of a local anesthetic between the fascia iliaca and the femoral nerve through an 18-gauge Tuohy needle at the inguinal crease level. FL: fascia lata, FA: femoral artery, FI: fascia iliaca, FN: femoral nerve. ${ }^{*}$ Local anesthetic. visualized on real-time color Doppler US.

After placement of the catheter, CFNB was initiated with 300 $\mathrm{ml}$ of $0.2 \%$ ropivacaine, infused continuously at a basal rate of 4 $\mathrm{ml} / \mathrm{h}$ with bolus injections of $2 \mathrm{ml}$ and a lockout time $15 \mathrm{~min}$ using a disposable infuser (Accufuser Plus ${ }^{\circledR}$, Wooyoung Medical, Seoul, Korea). Rescue analgesia with pethidine was available to all patients. The femoral hematoma, femoral abscess, or neurologic complications, including paresthesia or neurologic deficits, were observed at hospital room and orthopedic outpatient clinic during the 8-week follow-up period.

\section{Results}

Table 1 presents the demographic characteristics and perioperative data of all patients. Table 2 documents block outcomes and complications related to the US-guided femoral catheter insertions. The femoral catheters were placed with a $100 \%$ success rate. In 488 (100\%) patients, real-time US imaging revealed

Table 1. Patient Demographic Characteristics and Perioperative Data

\begin{tabular}{lc}
\hline & $\mathrm{N}=488$ \\
\hline Age (yr) & $68 \pm 11$ \\
Sex (M/F) & $107 / 381$ \\
Weight (kg) & $62 \pm 11$ \\
Height (cm) & $156 \pm 9$ \\
ASA physical status (I/II/III) & $51 / 334 / 103$ \\
Duration of surgery (min) & $84 \pm 13$ \\
Block procedure time (min) & $13 \pm 2$ \\
\hline
\end{tabular}

Values are expressed as mean \pm SD or number of patients. The block procedure time is the sum of the imaging, needling, and catheter placement times. ASA: American Society of Anesthesiologists.

Table 2. Block Outcome and Complications of Ultrasound-guided Femoral Catheter Placement in Anesthetized Patients

\begin{tabular}{lc}
\hline & $\mathrm{N}=488$ \\
\hline Success rate of catheter placement & $488(100 \%)$ \\
Separation of fascia iliaca and femoral nerve & $488(100 \%)$ \\
after LA injection (= confirmation of success) & \\
Catheter placement (easy/difficult) & $435(89 \%) / 53(11 \%)$ \\
Confirmation of the position of the catheter tip & $384(79 \%)$ \\
by real-time ultrasound & \\
VNRS pain score (0-10) at postoperative time point & \\
$1 \mathrm{~h}$ & $2.0 \pm 1.2$ \\
$6 \mathrm{~h}$ & $3.5 \pm 1.9$ \\
$12 \mathrm{~h}$ & $3.2 \pm 1.7$ \\
$24 \mathrm{~h}$ & $2.9 \pm 1.3$ \\
$48 \mathrm{~h}$ & $2.5 \pm 1.1$ \\
Inadvertent catheter removal & $14(3 \%)$ \\
Femoral artery puncture & $0(0 \%)$ \\
Small vascular puncture & $16(3 \%)$ \\
Femoral abscess & $0(0 \%)$ \\
Neurologic complications (during 8 weeks of follow up) & $0(0 \%)$ \\
\hline
\end{tabular}

Values are expressed as mean \pm SD or number of patients (\%). LA: local anesthetic, VNRS: verbal numerical rating scale. 
easy separation of the fascia iliaca and the femoral nerve following injection of a local anesthetic through the Tuohy needle. In 53 (11\%) patients, mild resistance was met during catheter insertion. In such cases, the bevel of the Tuohy needle was rotated, and this resulted in easy catheter insertion. In 384 (79\%) patients, the position of the catheter tip was visually confirmed on real-time color Doppler US via the spread of normal saline through the catheter near the femoral nerve.

Verbal numerical rating scale (VNRS) pain scores (0-10) were $2.0 \pm 1.2,3.5 \pm 1.9,3.2 \pm 1.7,2.9 \pm 1.3$, and $2.5 \pm 1.1$ at $1-, 6-, 12-, 24-$ and $48-\mathrm{h}$ postoperatively. VNRS pain scores are presented as mean \pm standard deviation. No femoral hematoma, femoral abscess, or neurologic complications (including paresthesia or neurologic deficits) were detected over the course of the 8-week follow-up period in the orthopedic outpatient clinic. In $16(3 \%)$ patients, a small volume of venous blood was aspirated through the femoral catheter. In these patients, compression lasting a few minutes was sufficient to stop the bleeding.

\section{Discussion}

In this retrospective study, femoral catheters were successfully placed in $100 \%$ of patients, without major complications. This success was confirmed through real-time US, which was used to visualize fascia iliaca and femoral nerve separation after local anesthetic injection through a Tuohy needle. There were no femoral hematoma, femoral abscess, and neurologic complications such as paresthesia or neurologic deficit during 8 weeks follow-up in 488 patients. This result suggests that our in-plane three-step needle insertion technique may be an effective and safe method for femoral catheter placement in anesthetized patients.

Nerve injury associated with peripheral nerve block is a relatively uncommon complication, and permanent injury is rare $[8,9]$. Considering that US was not used in many of the earlier studies on peripheral nerve blocks, it is difficult to compare these results to techniques that use real-time US guidance. It has been postulated that real-time needle-nerve visualization during US-guided peripheral nerve blocks reduces the risk of neurologic or vascular complications by preventing intraneural needle placement [7]. US provides the ability to dynamically visualize needle placement in order to reduce the risk of nerve puncture and intraneural injection of local anesthetic, and this may offer a greater safety margin for avoiding neurologic injury during peripheral nerve blocks. However, even under US guidance, peripheral nerve blocks may result in unintentional intraneural placement [10]. For example, Schafhalter-Zoppoth et al. [11] have reported inadvertent femoral nerve impalements and intraneural injection of local anesthetic during US-guided femoral nerve block. If the needle is advanced at a steep angle through the fascia iliaca toward the femoral nerve during conventional anatomy-based, nerve stimulation-based, or US-guided outof-plane needle insertion techniques, the tip of the needle can pierce the femoral nerve after penetrating the fascia iliaca, and this can result in intraneural injection or injury to the femoral nerve.

Therefore, we used an in-plane three-step needle insertion technique that was devised to reduce the risk of direct femoral nerve injury. In the first step, it is very important that the insertion point of the Tuohy needle is at the lateral part of the femoral nerve, thus preventing direct femoral nerve injury by the needle tip. Additionally, separation of the fascia iliaca and femoral nerve after injection of local anesthetic through the Tuohy needle is important for safe positioning of the needle shaft on the anterior surface of the femoral nerve. In the present study, easy separation of the fascia iliaca and the femoral nerve after local anesthetic injection through the needle was achieved in 488 (100\%) patients, and it was visualized by real-time US imaging.

Femoral catheters are usually inserted preoperatively and, to our knowledge, there have been no prospective, randomized controlled studies that have compared the relative risks of nerve injury from peripheral nerve blocks in anesthetized vs. non-anesthetized patients. For example, Watts and Sharma [12] analyzed 1,065 peripheral nerve blocks during a prospective 12-month follow-up study in which $45 \%$ of the blocks were performed under general anesthesia. A total of 13 patients had neurologic complications, only two of whom had blocks performed under anesthesia. Alternatively, Sawyer et al. [13] reported a background incidence of nerve palsy in $1 / 1,000$ (0.1\%) blocks performed under general anesthesia alone.

In this study, femoral catheters were inserted postoperatively in anesthetized patients to decrease patient discomfort and to reduce impact on the operating list. Paresthesia or pain is important in detecting nerve injury when CFNB is performed before anesthesia, as muscular contractions are elicited by nerve stimulation. In our study, contraction of the quadriceps femoris or sartorius muscle was still elicited in most patients because CFNB was performed after surgery, when muscular contraction power was nearly recovered in anesthetized patients. In patients who did not exhibit muscle twitching on nerve stimulation, correct placement of the Tuohy needle was confirmed via real-time US by visualizing the separation of fascia iliaca and the femoral nerve after injection of local anesthetic.

Our technique provided easy placement of the Tuohy needle on the anterior surface of the femoral nerve and insertion of the catheter through the Tuohy needle without difficulty in 435 (89.1\%) patients. The position of the catheter tip was confirmed on real-time color Doppler US via the spread of normal saline through the catheter near the femoral nerve (384 [79\%] patients). In the present study, we inserted the catheter tip at the 
anterior part of the femoral nerve, as conformation of successful Tuohy needle and catheter placement was easy, and the spread of the local anesthetic from the anterior part to the posterior part of the femoral nerve was effective.

There were some limitations to this study. First, this was a retrospective study. Thus, future studies should be prospective and should be conducted using detailed and objective evaluations of the neurologic complications and outcomes that result from procedures employing the in-plane, three-step needle insertion technique. Second, all blocks were performed by one anesthesiologist, which could be an issue when generalizing the results of this study.

In conclusion, this study revealed that our in-plane threestep needle insertion technique allowed for effective and easy placement of femoral catheters for TKA and resulted in no paresthesia or neurologic deficits in the 488 anesthetized patients evaluated in this study.

\section{References}

1. Salinas FV, Liu SS, Mulroy MF. The effect of single-injection femoral nerve block versus continuous femoral nerve block after total knee arthroplasty on hospital length of stay and long-term functional recovery within an established clinical pathway. Anesth Analg 2006; 102: 1234-9.

2. Ilfeld BM, Le LT, Meyer RS, Mariano ER, Vandenborne K, Duncan PW, et al. Ambulatory continuous femoral nerve blocks decrease time to discharge readiness after tricompartment total knee arthroplasty: a randomized, triple-masked, placebo-controlled study. Anesthesiology 2008; 108: 703-13.

3. Chelly JE. Femoral block. In: Peripheral nerve blocks: a color atlas. 3rd ed. Philadelphia, Wolters Kluwer Health/Lippincott Williams \& Wilkins. 2009, pp 1-496.

4. Fischer HB. Regional anaesthesia--before or after general anaesthesia? Anaesthesia 1998; 53: 727-9.

5. Oberndorfer U, Marhofer P, Bösenberg A, Willschke H, Felfernig M, Weintraud M, et al. Ultrasonographic guidance for sciatic and femoral nerve blocks in children. Br J Anaesth 2007; 98: 797-801.

6. Ponde V, Desai AP, Shah D. Comparison of success rate of ultrasound-guided sciatic and femoral nerve block and neurostimulation in children with arthrogryposis multiplex congenita: a randomized clinical trial. Paediatr Anaesth 2013; 23: 74-8.

7. Marhofer P, Chan VW. Ultrasound-guided regional anesthesia: current concepts and future trends. Anesth Analg 2007; 104: 1265-9.

8. Brull R, McCartney CJ, Chan VW, El-Beheiry H. Neurological complications after regional anesthesia: contemporary estimates of risk. Anesth Analg 2007; 104: 965-74

9. Barrington MJ, Watts SA, Gledhill SR, Thomas RD, Said SA, Snyder GL, et al. Preliminary results of the Australasian Regional Anaesthesia Collaboration: a prospective audit of more than 7000 peripheral nerve and plexus blocks for neurologic and other complications. Reg Anesth Pain Med 2009; 34: 534-41.

10. Bigeleisen PE. Nerve puncture and apparent intraneural injection during ultrasound-guided axillary block does not invariably result in neurologic injury. Anesthesiology 2006; 105: 779-83.

11. Schafhalter-Zoppoth I, Zeitz ID, Gray AT. Inadvertent femoral nerve impalement and intraneural injection visualized by ultrasound. Anesth Analg 2004; 99: 627-8.

12. Watts SA, Sharma DJ. Long-term neurological complications associated with surgery and peripheral nerve blockade: outcomes after 1065 consecutive blocks. Anaesth Intensive Care 2007; 35: 24-31.

13. Sawyer RJ, Richmond MN, Hickey JD, Jarrratt JA. Peripheral nerve injuries associated with anaesthesia. Anaesthesia 2000; 55: 980-91. 